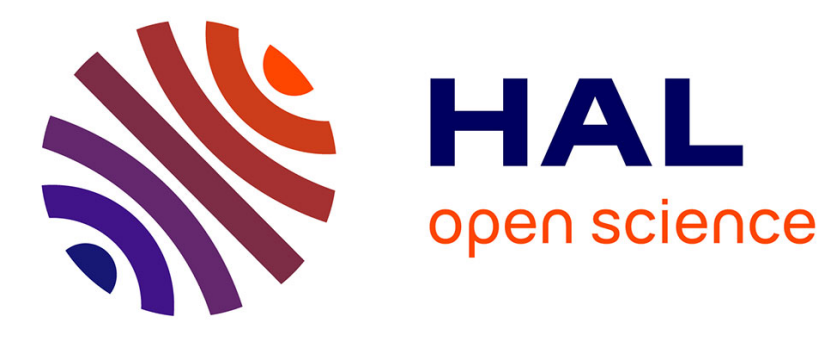

\title{
Results of Coring from a Low Mass Rover
}

\author{
Paul Backes, Daniel Helmick, Max Bajracharya, Oussama Khatib, Vincent \\ Padois, James Warren
}

\section{To cite this version:}

Paul Backes, Daniel Helmick, Max Bajracharya, Oussama Khatib, Vincent Padois, et al.. Results of Coring from a Low Mass Rover. IEEE Aerospace Conference, Mar 2008, Big Sky, United States. pp.1-7, 10.1109/AERO.2008.4526238 . hal-00624328

\section{HAL Id: hal-00624328 \\ https://hal.science/hal-00624328}

Submitted on 16 Sep 2011

HAL is a multi-disciplinary open access archive for the deposit and dissemination of scientific research documents, whether they are published or not. The documents may come from teaching and research institutions in France or abroad, or from public or private research centers.
L'archive ouverte pluridisciplinaire HAL, est destinée au dépôt et à la diffusion de documents scientifiques de niveau recherche, publiés ou non, émanant des établissements d'enseignement et de recherche français ou étrangers, des laboratoires publics ou privés. 


\section{Results of Coring from a Low Mass Rover}

\author{
Paul Backes, Daniel Helmick, Max Bajracharya \\ Jet Propulsion Laboratory \\ California Institute of Technology \\ Paul.G.Backes@jpl.nasa.gov
}

\author{
Oussama Khatib, Vincent Padois, and James Warren \\ Stanford University \\ khatib@es.stanford.edu
}

\begin{abstract}
Technology for coring from a low-mass rover has been developed to enable core sample acquisition where a planetary rover experiences moderate slip during the coring operation. A new stereo vision technique, Absolute Motion Visual Odometry, is used to measure rover slip during coring and the slip is accommodated through corresponding arm pose updating. Coring rate is controlled by feedback of the measured force of the coring tool against the environment. Test results in the JPL Marsyard show that coring from a low-mass rover with slip is feasible. ${ }^{12}$
\end{abstract}

\section{TABLE OF CONTENTS}

1. INTRODUCTION. 1

2. ABSOlUte Motion Visual OdOMETRY..............2

3. COORDINATED ROVER-ARM CONTROL ................2

4. ROVER INITIAL POSE ............................................

5. IMPLEMENTATION ENVIRONMENT ........................ 4

6. RESULTS ............................................................ 4

7. CONCLUSION AND FUTURE WORK .....................6

ACKNOWLEDGEMENTS ........................................... 6

REFERENCES ...................................................... 6

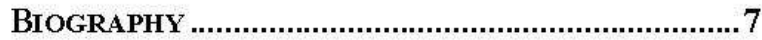

\section{INTRODUCTION}

Technology to enable core sample acquisition from a lowmass rover with modest slip during coring has been developed. The current state-of-the-art in coring and drilling from a planetary rover arm-mounted tool, represented by the upcoming 2009 Mars Science Laboratory (MSL) mission, assumes that the arm and rover act as a rigid platform for the coring tool or drill. The MSL mission rover will have a drill mounted on a manipulator. The manipulator will anchor the drill against a rock by pressing tines against the rock to provide a rigid base for the drill. The drill will then provide its own translational degree-offreedom (DOF) and control of interaction with a rock. It is reasonable to assume that the rover will not move during the coring operation if the rover is big enough, as is the case for the MSL mission, but this assumption is not reasonable when the rover's mass is low relative to the interaction forces with the environment or when the rover is on a slope where slippage of the rover is possible.

Future missions could benefit by enabling arm-mounted drilling or coring from a low-mass rover where the rover

\footnotetext{
${ }_{1}^{1} 1-4244-1488-1 / 08 / \$ 25.0002008$ IEEE

${ }^{2}$ IEEEAC paper \#1122, Version 2, Updated December 21, 2007
}

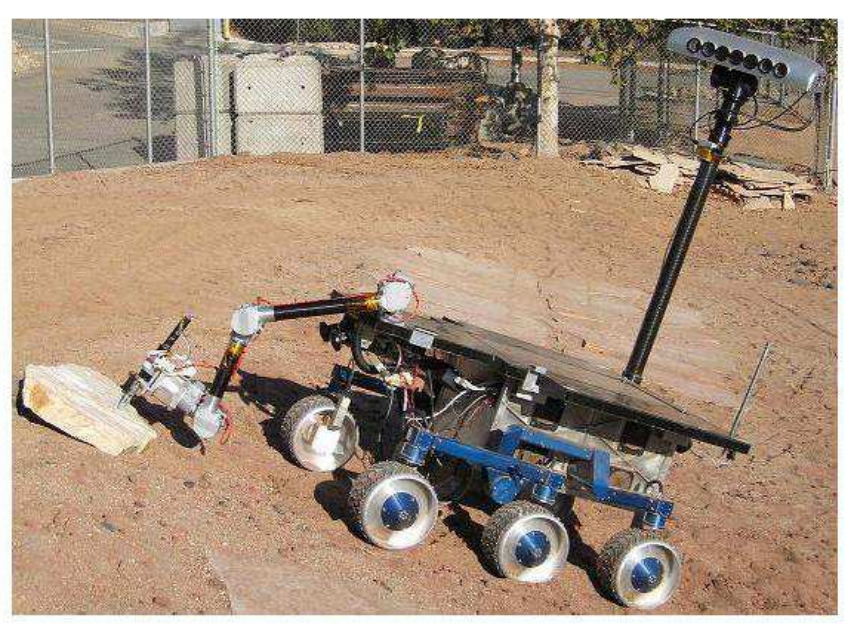

Figure 1: Coring on a 26 degree slope in the Marsyard

may experience modest slippage during the drilling or coring operation. A low-mass rover might be on the order of $100-200 \mathrm{~kg}$. A future Mars Sample Return (MSR) mission might utilize a low-mass rover that acquires core samples that are stored and returned to Earth [1][2]. It is desirable to eliminate the need to provide rigid contact with the environment via pushing against the environment with tines and instead allow the manipulator to provide positioning of the coring tool relative to the environment. This could allow for a lower mass system and allow sensing of the tool-environment interaction to be done via sensors on the arm such as motor currents or torque sensing. To enable coring from a low-mass rover, NASA's Mars Technology Program sponsored the work described here.

Figure 1 illustrates the problem that we are trying to solve. A low-mass rover has a coring tool at the end of a five DOF arm and we need to acquire a core sample with the rover on a 26 degree slope. Soil models indicate that a low rate of rover slip can be expected during the coring operation due to the vibration from the coring action. This rate of slip will be small relative to the control bandwidth of the arm, so if the slippage can be measured, then it should be possible to reconfigure the arm to keep the tool aligned with the coring hole during the coring operation.

The overall approach for coordinated rover and arm motion to accommodate slip of the rover during a coring operation is to measure the rover slip and reconfigure the arm so that the tool remains aligned with the coring hole. If the rover slips far enough so that the arm reaches the edge of its 
workspace, then the rover is driven so that the arm is in the middle of its workspace with the coring bit in the hole. During this process the coring bit is not removed from the coring hole. There might be scenarios where removing the bit and reinserting it might be feasible, but it is preferred to find a solution where the bit remains in the hole until the sample has been acquired. In our approach a stereo vision technique, Absolution Motion Visual Odometry (AMVO), described here for the first time, is used to measure the rover motion, from slip or driving, relative to its initial pose. This motion is accommodated by reconfiguration of the arm to keep the tool aligned with the coring hole. Single axis force control is used for motion of the tool along the tool axis in the hole during coring. Tool rotational velocity is used to measure binding as a safety feature. It was decided to not use six-axis force sensing and control of the tool in the hole because there is no flight qualified six axis force-torque sensor available for use. The approach described here enables coring with modest slip without the use of tines and using existing planetary rover manipulation and sensing capabilities.

\section{Absolute Motion Visual Odometry}

We developed Absolute Motion Visual Odometry (AMVO) to measure the six degrees of freedom (DOF) of motion of a rover for small motions of the vehicle (less than about 50 $\mathrm{cm})$. We use this technique to accurately detect and measure the slippage that a rover undergoes while coring a sample from a rock in its environment. AMVO uses the same algorithm as visual odometry (VO) [3][4][5][6][7][8] but it uses the concept of a key pair to avoid the measurement drift associated with VO.

Stereo camera motion estimation, or visual odometry, is used to sense the $6 \mathrm{DOF}$ rover motion due to slip during the course of coring activity. This 6 DOF of motion is represented by a transform that is used in a transform equation resulting in an updated arm configuration to keep the tool at the original pose (and then offset along the coring axis for the coring operation). Because only small motions need to be estimated, standard camera motion estimation methods are modified to use a key frame (image pair) taken before coring. All subsequent frames are then compared to this key frame and thus avoid the accumulation of error between successive frames. Because all motion is measured with respect to a single fixed frame, we term this method Absolute Motion Visual Odometry.

In order to use AMVO to compensate for rover motion during coring, it must be able to detect very small motions and run fast enough to be used in the real-time closed-loop system. Two implementations of AMVO were evaluated. The first was the more common technique of detecting, tracking, and robustly matching feature points [3][4][6][8]. The second implementation makes use of Euclidean constraints to select inliers and avoid tracking features [5]. Both techniques begin by autonomously selecting features in the first frame, matching them between left and right camera images, and triangulating their $3 \mathrm{D}$ positions. The first implementation then uses a correlation method to track the features to the next frame and triangulate the new positions, and then uses a rigidity test to reject outliers. Finally, this approach uses a least median squares with a weighted least squares matching method to robustly choose an initial motion estimation, and then refines this estimate with a non-linear maximum likelihood procedure that incorporates the stereo error covariances directly into the estimation. The second method does not track features, but rather detects features in the next frame, uses the SAD (sum of absolute difference) score from stereo matching to generate initial feature matches, and then refines the matches using geometric (point rigidity and angular motion) constraints. The motion of the matched points is then calculated using a non-linear optimization of image-space error. As a result, this algorithm can be implemented faster, but could suffer from some reduction in accuracy.

We found that both VO methods performed similarly for computing absolute motion, although the second (feature matching rather than tracking) could be implemented to be slightly faster (as expected). Both methods were able to detect $0.5 \mathrm{~mm}$ motions, and could achieve better than $2 \mathrm{~mm}$ accuracy for $4 \mathrm{~mm}$ to $20 \mathrm{~cm}$ motions in realistic outdoor Mars-like environments (more precise measurements could not be made due to limitations of our outdoor groundtruthing equipment). The full 6 DOF motion of the vehicle was ground-truthed by measuring three rover-mounted fiducials with an outdoor laser surveying instrument. Motion magnitudes ranging from $4 \mathrm{~mm}$ to $20 \mathrm{~cm}$ were measured, and visual odometry was performed on the vehicle's $1024 \times 786$ (but makes use of image pyramiding) grayscale body-mounted "hazcams", which have an approximately 110 degree diagonal field-of-view. On the onboard 1.2GHz Pentium M CPU, our implementation of the first algorithm can be run at approximately $3 \mathrm{~Hz}$, whereas the second can be run at approximately $5 \mathrm{~Hz}$. Since the two algorithms produced similar results but the second algorithm was slightly faster, we used the second algorithm in our implementation of AMVO.

Results of AMVO in a small motion test are shown in Figure 2. The rover was commanded with the smallest possible motion and AMVO was used to measure the motion. This resulted in a measured motion of $0.54 \mathrm{~mm}$.

\section{Coordinated Rover-Arm Control}

The arm motion to push the coring tool into the hole and accommodate rover slippage was controlled at $20 \mathrm{~Hz}$ with the arm command computed using Equations (1) and (2). 


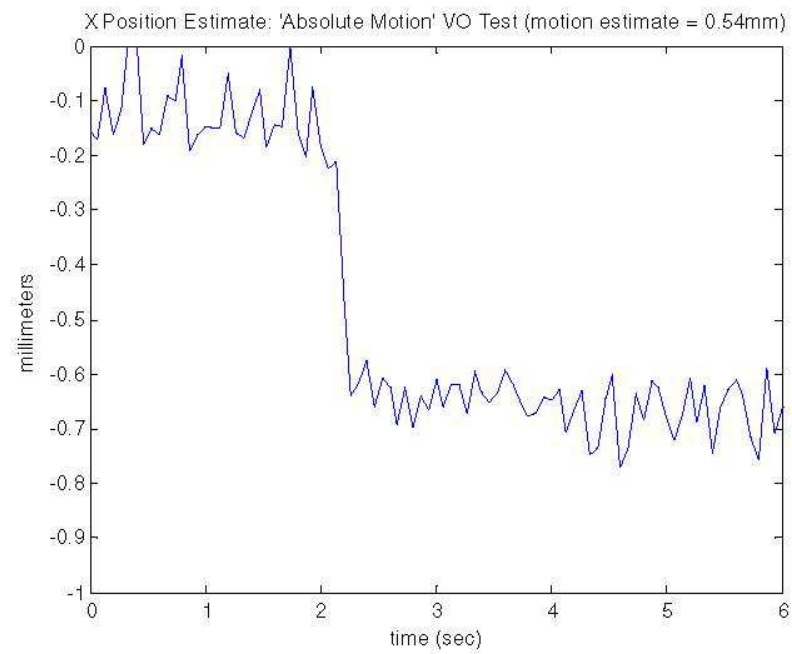

Figure 2: AMVO small motion test

$$
\begin{aligned}
& { }_{T C}^{R C} T(i)={ }_{R i}^{R C} T(i) *{ }_{T i}^{R i} T *{ }_{T C}^{T i} T(i) \\
& { }_{T C}^{T i} T(i)={ }_{T C}^{T i} T(i-1) * *^{T C} T_{S}(i)
\end{aligned}
$$

${ }_{R i}^{R c} T(i)$ is the $6 \mathrm{DOF}$ transform from the Rover frame initial pose where the coring operation started to the Rover frame current pose. This transform is computed by AMVO at each sample interval. ${ }_{T i}^{R i} T$ is the transform from the Tool frame to the Rover frame at the start of the coring operation and is a constant. ${ }_{T C}^{T i} T(i)$ is the transform from the Tool frame current commanded pose to the Tool frame initial pose when coring was started. ${ }^{{ }^{C}} T_{S}(i)$ is the transform that contains the sensor based motion command generated in the current sample interval. For coring motion, ${ }^{{ }^{T c}} T_{S}(i)$ has only a tool translation axis ( $\mathrm{Z}$ axis) motion, $\Delta z$, generated from force control. $\Delta z$ is computed with Equation (3)

$$
\Delta z=K_{f} *\left(f_{z d}-f_{z a}\right)
$$

Where $\left(f_{z d}-f_{z a}\right)$ is the difference between the desired average weight on bit of the coring tool and the measured average weight on bit, and $K_{f}$ is the control gain. ${ }_{T C}^{R C} T(i)$ is computed each sample interval and then used in inverse kinematics to compute arm joint angles which are then commanded and controlled.

When the rover moves so the arm reaches the edge of its workspace, the rover is driven back to its pose at the start of coring to put the arm back in the center of its workspace. The rover motion is controlled in a separate process from the arm control so the arm control based on AMVO is used to autonomously accommodate the rover motion without any prior knowledge of the planned rover motion. And coring continues during this rover drive to reset the rover pose.

\section{Rover Initial Pose}

During a Mars mission a rover might approach a coring target from below, as is done in the tests shown here, or from other orientations such as transverse of the face of the slope. For any approach orientation, the rover's pose relative to the target needs to be computed. One approach assumes an approach direction defined by the geometry of the target and computes the rover position along that approach direction that maximizes arm manipulability [9]. An alternative approach has been developed that considers the rover mobility characteristics, wheel-soil force distribution, and the rover arm's ability to apply forces. This has been implemented and tested within the Stanford Simulation and Active Interface (SAI) system [10]. SAI is unique in that it is a real-time interactive environment that allows the user to apply and sense forces within the virtual world via haptic devices. SAI features multi-contact resolution for multi-body systems, efficient algorithms for articulated body dynamics, and simulated friction and ground reaction forces. SAI is based upon a general framework [11] for the resolution of multi-contact between articulated multi-body systems in the context of operational space control for robots [12]. Using this framework, the dynamic relationships between all existing contact points can be described. These relationships are characterized by the masses as perceived at the contact points. A force exerted at a contact point, whether from a collision with another object or from interaction with a user, can be translated into forces at all related contact points. The necessary computations are performed with an efficient recursive algorithm. The contact space representation allows interaction between groups of dynamic systems to be described easily without having to examine the complex equations of motion of each individual system. A collision model can be developed with the same ease as if one were considering interaction only between simple bodies. Impact and contact forces between interacting bodies can then be solved efficiently. The rover pose algorithm computes the optimal coring configuration given the coring conditions: terrain slope, coring point location, and ground type. This requires the definition of the criterion used to characterize the optimality of a given configuration. We used different manipulability measures [13] so that the force capabilities of the robot along the coring tool axis are maximized and the overall manipulability is maximized in all operational directions. 


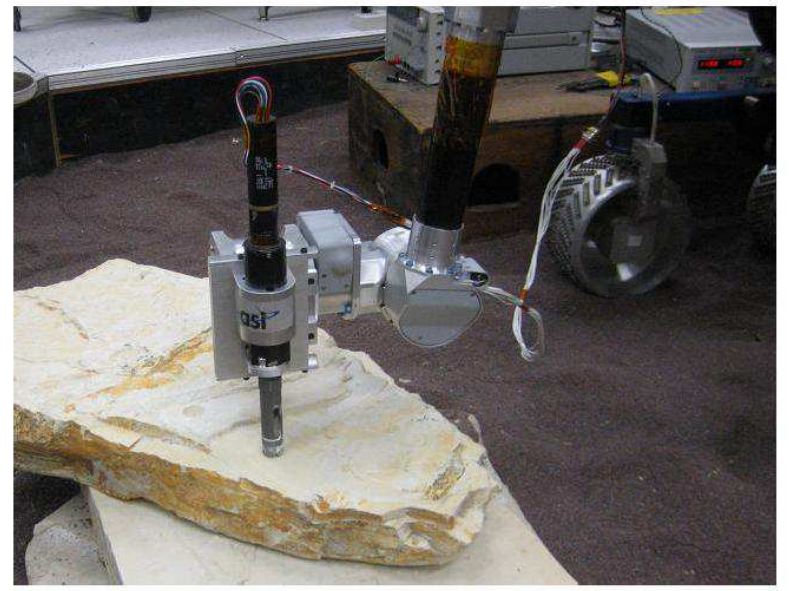

Figure 3: Percussive coring test

\section{IMPLEMENTATION ENVIRONMENT}

A coring tool was attached to the arm on a $65 \mathrm{~kg}$ six wheel rocker-bogie rover called Pluto, shown in Figure 1, and coring tests were performed. The Pluto rover has the same mechanical mobility system as the FIDO rover [14] but has a different mast and avionics system and has a five DOF manipulator. The avionics system has a $1.2 \mathrm{GHz}$ Pentium M $\mathrm{CPU}$ running Linux $\mathrm{O} / \mathrm{S}$. Arm and wheel motor control is done using ION commercial motor controllers from Performance Motion Devices, Inc. The coring tool is the LSAS coring tool from Alliance Spacesystems [15]. The LSAS tool is a rotary percussive coring tool weighing 400 grams and requires about $15 \mathrm{~N}$ average weight on bit. It rotates at $800 \mathrm{RPM}$ with 3 hits per revolution, and requiring about 20 Watts.

\section{RESULTS}

The objective of this work was to develop the technology needed to demonstrate that coring from a low-mass rover is feasible for future Mars rover missions. The first step was to find a coring tool that represents the functionality of the coring tool that would be on such a mission. It was concluded that a rotary percussive coring tool would be used because a percussive coring tool requires significantly lower weight on bit than a rotary friction coring tool. Since a representative percussive coring tool had never been used to acquire a core from a rover previously, an LSAS coring tool was mounted to the end of the Pluto rover five DOF arm and used to acquire a core from limestone, as shown in Figure 3.

Figure 4 shows the bit used in the coring test, the hole, and the resulting core. The results were significant in demonstrating for the first time the feasibility of core sample acquisition with such a device. The first significant

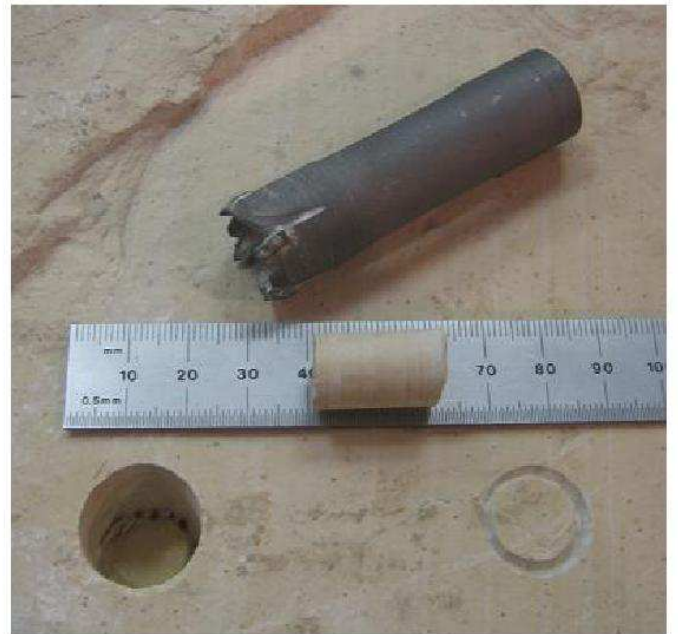

Figure 4: Core and bit from percussive coring test

result was that the core started without tines or a centering bit. The core started without a centering bit or tines because with a percussive tool the bit rebounds from the surface after each impact and returns to a nominal position. Disturbance forces that would cause the tool to skitter (move away from the coring position) are accommodated by the stiffness of the arm. The core was acquired at a rate of 2 $\mathrm{cm} /$ hour in limestone. Cuttings were artificially removed using a vacuum. Cuttings removal is a critical capability for coring tools and is not available in this first version of the tool. The test was stopped after one hour. The core was removed from the hole by inserting a thin metal rod down the side of the core and breaking it off. Core breakoff is another capability that needs to be added to such a device for planetary applications. The core was $2 \mathrm{~cm}$ long with constant $12 \mathrm{~mm}$ diameter along its length. A longer core could have been acquired in a longer coring operation. This rate of coring, about $2 \mathrm{~cm} /$ hour, is representative of the

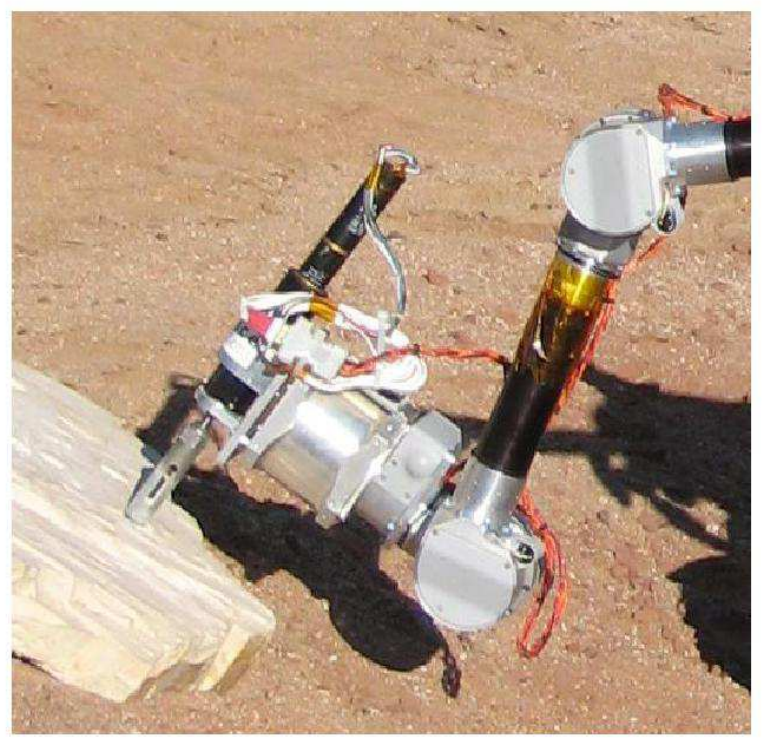

Figure 5: Coring on a slope in the Marsyard 


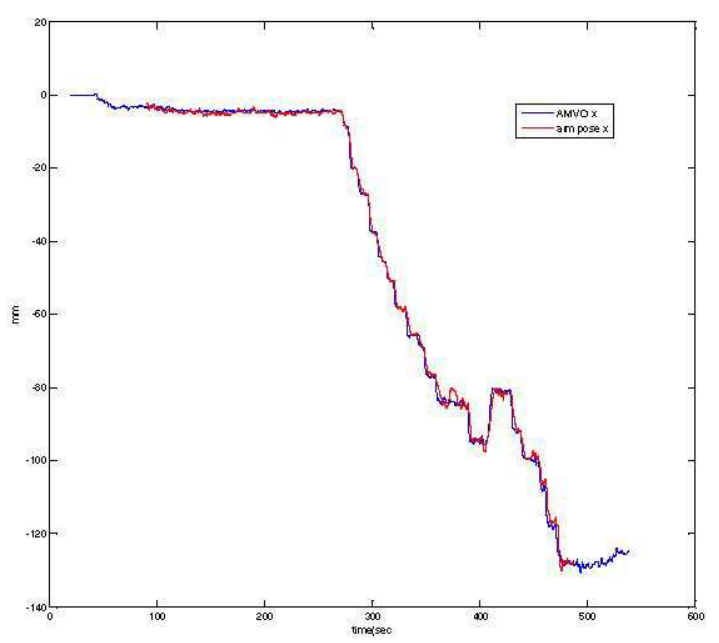

\section{Figure 6: AMVO output and arm motion during whole coring operation}

expected rate of coring for Mars mission applications. A wrist force-torque sensor was used to measure the maximum impact forces felt by the arm during the coring operation, and were measured to be about $60 \mathrm{~N}$. The core quality was a second significant result. Coring attempts with other tools had resulted in broken cores. The low average weight on bit, $15 \mathrm{~N}$, was also significant because that is an achievable applied force for a low-mass rover.

Coring on a slope with slip is shown in Figure 1 and Figure 5. The rover was placed on a 26 degree slope in the JPL Marsyard and the tool was deployed to a limestone rock for coring. The AMVO control technique was used to keep the tool aligned with the coring target while the rover moved during the coring operation. Rover slip when starting the

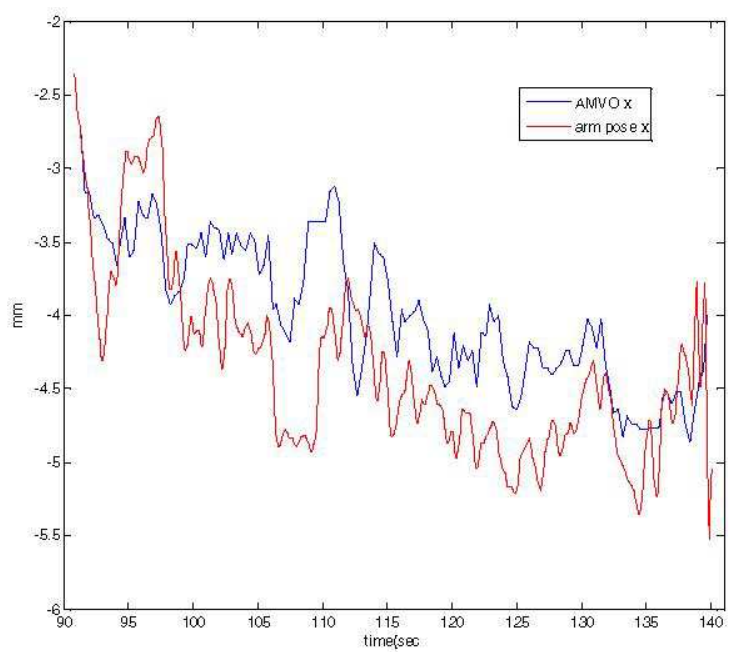

Figure 7: AMVO output and arm position at start

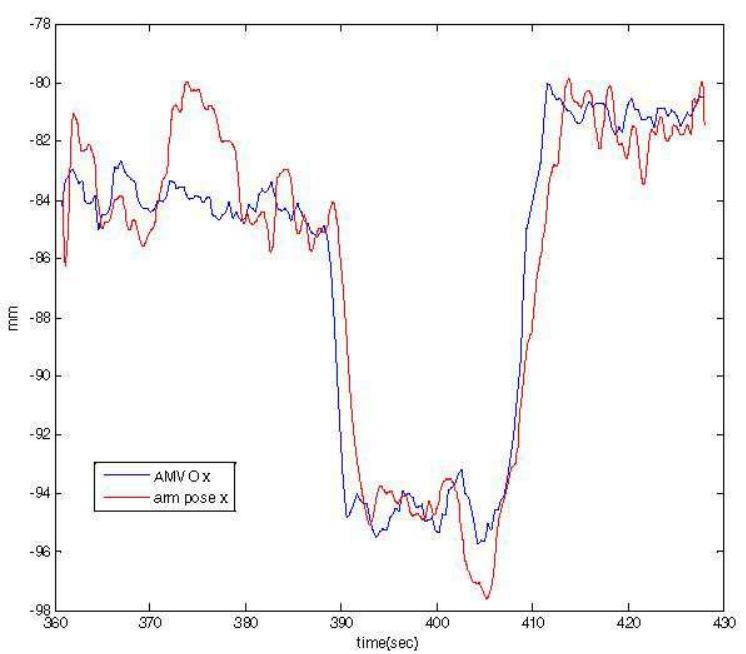

Figure 8: AMVO output and arm position during drives

core and during the coring operation were autonomously accommodated by updating the arm pose based on AMVO feedback as described in section 3 . Figures 6,7 , and 8 show the AMVO output in the Rover frame X direction (forward) and the measured arm Tool frame position in the same direction (changed sign so that they would overlap; the actual motions are in opposite directions) during the coring operation. The coring tool was turned on just before contacting the rock. The core was autonomously started and the coring operation continued for about 260 seconds. Then, in order to show robustness of the system to larger rover motions, the rover was purposely driven down the hill in steps of about $1 \mathrm{~cm}$. The arm accommodation using AMVO kept the coring tool safely in the coring hole and continuing to core. Then to demonstrate driving the rover back up the hill to put the arm closer to the center of its workspace, it was driven up about $1.5 \mathrm{~cm}$. Then it was driven down to the limit of about $13 \mathrm{~cm}$ from the starting point. The arm accommodated all motions, keeping the tool safely coring in the hole. Figure 6 shows the data over the whole motion where the rover moved $13 \mathrm{~cm}$ down the hill during the coring operation. Figure 7 shows the motion at the start of the coring operation. As expected, there is a small amount of slip by the rover (about $1.5 \mathrm{~mm}$ over 50 seconds), AMVO measures the slip motion, and the arm is commanded to change configuration to accommodate the slip motion. Figure 8 shows the AMVO output and arm motion when the rover was driven down and then up the hill at about $390-410$ seconds. There is a slight lag in the arm motion to accommodate the AMVO outputs as expected. Figure 9 shows a back hazcam image used by AMVO during the coring operation. The results show that use of AMVO to generate arm commands works over the $13 \mathrm{~cm}$ of motion. 


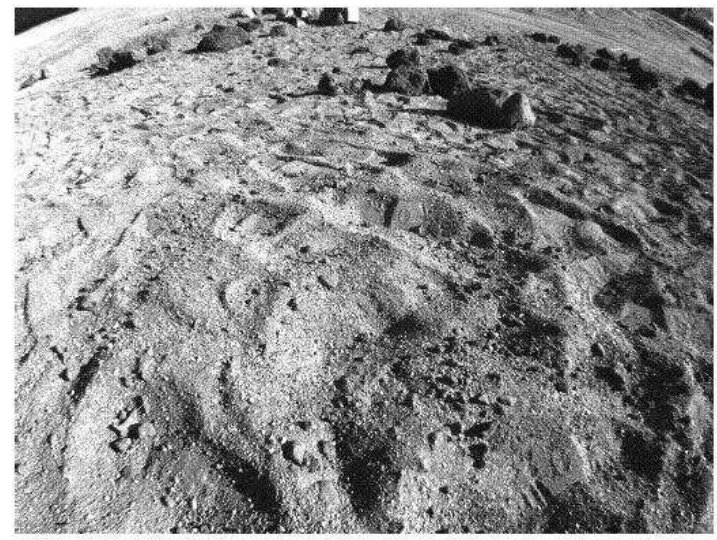

Figure 9: Back hazcam image used by AMVO

\section{CONClusion AND Future Work}

Technologies to enable coring from a low-mass rover for planetary applications were developed and demonstrated including starting a core without a centering bit or stabilizing tines, coring with low weight on bit, and coring from a rover that is experiencing slip. Absolute motion visual odometry was developed and shown to provide sufficiently accurate measurement of rover motion during the coring operation to be used to compute the arm accommodation to keep the coring tool in the coring hole. The tool motor rotational speed was found to be an indicator of the binding of the tool in the hole. We plan on integrating motor current and speed for fine adjustment of tool position to minimize friction between the tool and hole. Since acquiring a core can take hours, the shadows of features will change during this time, potentially affecting the accuracy of AMVO relative to the initial key frames. We will investigate the affect of changing shadows and compensate for the shadow affects. Separately, coring tool technology is being advanced to include required capabilities for planetary applications including core breakoff, core retention, core ejection, and bit changeout.

\section{ACKNOWLEDGEMENTS}

The research described in this publication was carried out at the Jet Propulsion Laboratory of California Institute of Technology and Stanford University under contract from the National Aeronautics and Space Administration (NASA), with funding from the Mars Technology Program, NASA Science Mission Directorate.

\section{REFERENCES}

[1] H. Price, K. Cramer, S. Doudrick, W. Lee, J. Matijevic, $\mathrm{S}$. Weinstein, T. Lam-Trong, $\mathrm{O}$. Marsal, and $\mathrm{R}$. Mitcheltree, "Mars Sample Return Spacecraft Systems
Architecture," 2000 IEEE Aerospace Conference Proceedings, pp. 357-375.

[2] R. Mattingly, S. Matousek, and F. Jordan, "Continuing Evolution of Mars Sample Return," 2004 IEEE Aerospace Conference Proceedings, paper \#1238.

[3] L. Matthies. Dynamic stereo vision. Ph.D. thesis, Carnegie Mellon University, October 1989.

[4] C. F. Olson, L.H. Matthies, M. Shoppers, and M. Maimone, Stereo ego-motion Improvements for robust rover navigation, In Proceedings of the IEEE International Conference on Robotics and Automation, pages 1099-1104, 2001.

[5] H. Hirschmuller, P.R. Innocent, J.M. Garibaldi. Fast, unconstrained camera motion estimation from stereo without tracking and robust statistics. Control, Automation, Robotics and Vision (ICARCV'02), pages 1099--1104, 2002.

[6] Y. Cheng, M. Maimone, and L. Matthies. Visual odometry on the Mars Exploration Rovers. IEEE International Conference on Systems, Man and Cybernetics, 2005.

[7] D. Helmick, S. Roumeliotis, Y. Cheng, D. Clouse, M. Bajracharya, and L. Matthies, "Slip-Compensated Path Following for Planetary Exploration Rovers," Journal of Advanced Robotics, Volume 20, Number 11, pp. 1257-1280, November, 2006.

[8] M. Maimone, Y. Cheng, and L. Matthies, "Two Years of Visual Odometry on the Mars Exploration Rovers," Journal of Field Robotics, Volume 24, Number 3, special issue on Space Robotics, March 2007, pp 169186.

[9] P. Backes, A. Diaz-Calderon, M. Robinson, M. Bajracharya, and D. Helmick, "Automated Rover Positioning and Instrument Placement," Proceedings IEEE Aerospace Conference, March 5-12, 2005.

[10] O. Khatib, O. Brock, K. Chang, F. Conti, D. Ruspini and L. Sentis, "Robotics and Interactive Simulation", Communications of the ACM, 45(3), 46-51, March 2002.

[11]D. Ruspini and O. Khatib, "Collision / Contact Models for Dynamic Simulations and Haptic Interaction", Proceedings of the International Symposium on Robotics Research, 185-195, 1999.

[12] O. Khatib, "A Unified Approach to Motion and Force Control of Robot Manipulators: The Operational Space Formulation", Int. J. Robot. Automa., 3 (1), 43-53, 1987.

[13]I. D. Walker, "Impact configurations and measures for kinematically redundant and multiple armed robot systems", IEEE Transactions on Robotics and Automation, 10(5), 670-683, 1994.

[14]P. S. Schenker, E. T. Baumgartner, L. I. Dorsky, P. G. Backes, H. Aghazarian, J. S. Norris, T. L. Huntsberger, Y. Cheng, A. Trebi-Ollennu, M. S. Garrett, B. A. Kennedy and A. J. Ganino,R. E. Arvidson,S. W. Squyres, "FIDO: a Field Integrated Design \& Operations rover for Mars surface exploration," Proc. 6th International Symposium on Artificial Intelligence, 
Robotics and Automation in Space (i-SAIRAS-'01), Montreal, Canada, June 18-21, 2001

[15]S. Stanley, S. Dougherty and J. Laramee, "The Lowforce Sample Acquisition System," 2007 NASA Science and Technology Conference Proceedings, June, 2007.

\section{BIOGRAPHY}

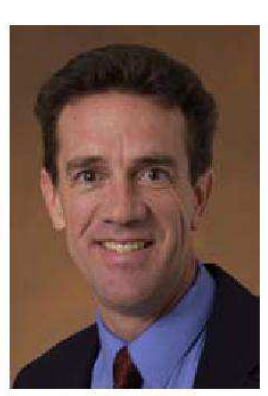

Paul Backes, Ph.D. is the Group Supervisor of the Mobility and Manipulation group at Jet Propulsion Laboratory, California Institute of Technology, where he has been since 1987. He received the BSME degree from U.C. Berkeley in 1982 and Ph.D. in 1987 in Mechanical Engineering from Purdue University. Dr. Backes received the 1993 NASA Exceptional Engineering Achievement Medal for his contributions to space telerobotics, 1998 JPL Award for Excellence, 1998 NASA Software of the Year Award Sole Runner-up, and 2004 NASA Software of the Year Award. He has served as an Associate Editor of the IEEE Robotics and Automation Society Magazine.

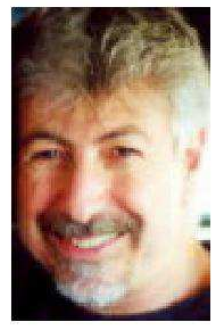

Oussama Khatib, Ph.D. is a Professor of Computer Science at Stanford University. He received his Ph.D. in 1980 from Sup'Aero, Toulouse, France. His current research is in human-centered robotics, human motion synthesis, humanoid robotics, dynamic simulation, haptic interfaces, and human-friendly robot design. This builds upon a large body of work pursued over the past 25 years and published in over 200 contributions in the field. Professor Khatib was Program Chair of ICRA2000 and editor of The Robotics Review. He served as director of the Stanford Computer Forum and is currently president of the International Foundation of Robotics Research and editor of Springer Tracts in Advanced Robotics. Professor Khatib is an IEEE Fellow, a Distinguished Lecturer of IEEE, and a recipient of the JARA Award.

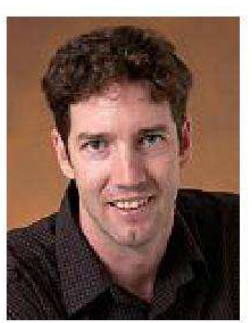

Daniel Helmick received his B.S degree in Mechanical Engineering from Virginia Polytechnic Institute and State University and his M.S. in Mechanical Engineering with a specialization in controls from Georgia Institute of Technology in 1996 and 1999 respectively. Since June 1999 he has been working at the Jet Propulsion Laboratory on robotics research projects involving vision/sensor based control of robots, state estimation, and navigation and mobility algorithms. He has worked on robotic vehicles covering a wide range of functionality, including: Mars research rovers for rough terrain mobility; small, tracked robots for urban mobility; a cryobot for ice penetration; and reconfigurable wheeled robots for Mars exploration. His research interests include: sensor-based control of robots, sensor fusion and state estimation, and rover navigation and mobility.

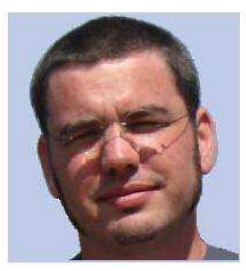

Vincent Padois, Ph.D. is an assistant professor of Computer Science and a member of the Institute for Intelligent Systems and Robotics at Pierre et Marie Curie University in Paris, France. He received a master's degree in 2001 and his Ph.D. in 2005 both in Automatic Control from the Toulouse National Polytechnic Institute. In 2006 and 2007, he was a postdoctoral fellow in the group of Professor O. Khatib at Stanford University. His research mainly focuses on the modeling and control of redundant and complex systems such as wheeled mobile manipulators and humanoid robots. He is also involved in the field of human motion synthesis using model learning techniques and in development of dynamic simulation and collision detection software.

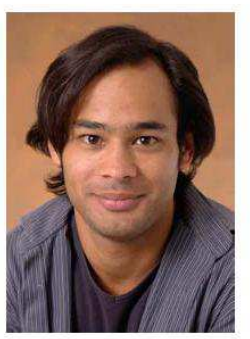

Max Bajracharya received $M$. Eng., B.S.,EE/CS degrees from MIT in 2001. $\mathrm{He}$ is a member of the Mobility and Manipulation Group at the Jet Propulsion Laboratory, Pasadena, $C A$. His research includes visionbased tracking, manipulation, navigation, and pose estimation for Mars rovers.

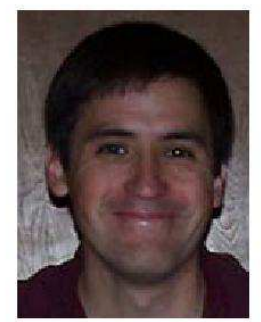

James Warren is a Ph.D. candidate in the Artificial Intelligence Laboratory at Stanford University. He received his B.S. degree in Mathematics from Texas A\&M University in 1996 and his M.S. degree in Scientific Computing and Computational Mathematics in 2000. His research is in identifying human motion behaviors from motion capture data and applying these strategies for humanoid robotic control. 